\section{Erfolgreiche Behandlung einer benignen Trachealstenose durch einen Silikonstent (Polyflex-Stent) über 5 Jahre}

J. Schildge ${ }^{1}$, H. Hauk ${ }^{2}$

${ }^{1}$ Medizinische Klinik Abteilung Pneumologie, St. VincentiusKrankenhäuser Karlsruhe (Chefarzt: Dr. J. Schildge)

2 Pathologisches Institut, St. Vincentius-Krankenhäuser Karlsruhe (Direktoren: Prof. Dr. H. Hauk, Prof. Dr. H.-M. Schneider)
Zusammenfassung: Inoperable, funktionell wirksame Stenosen des Tracheobronchialsystems können durch bronchoskopische Stenteinlage kurz- und mittelfristig oft effektiv behandelt werden. Silikonstents weisen bei langer Liegezeit gelegentlich Probleme in Form von Migration und Sekretretention auf. Metallstents können zu Granulationsgewebsbildung oder sogar Wandperforation führen. Hinsichtlich der Langzeitbehandlung mit Atemwegsstents über mehrere Jahre gibt es wenig Erfahrungen und keine einheitlichen Empfehlungen. Wir berichten über eine 76-jährige Patientin, die wegen einer benignen subglottischen Trachealstenose auf dem Boden zweier Tracheotomien mittels bronchoskopischer Dilatation und Einlage eines Silikon-Stents (POLYFLEX-Stent) behandelt wurde. Während einer Nachbeobachtungsdauer bislang von fast 5 Jahren kam es zu keiner Rezidivstenose. Schwere Stentkomplikationen in Form von Stentmigration, Sekretblockade und Granulationsgewebsbildung traten nicht auf. Eine Neigung zur bronchialen Verschleimung bot unter regelmäßiger Inhalationstherapie mit isotoner NaCl-Lösung keine Probleme. 21 und 56 Monate nach Stenteinlage kam es zu geringen Hämoptysen, ohne dass bronchoskopisch eine Blutungsquelle nachweisbar war. Histologisch war nach 56 Monaten am distalen Stentende eine Plattenepithelmetaplasie nachweisbar. Mikrobiologisch zeigte sich eine zunehmende, klinisch jedoch inapparente Besiedelung des Bronchialsekretes mit potenziell pathogenen Keimen.

Successful Treatment of a Benign Tracheostenosis with a Silicone Stent (Polyflex-Stent) over 5 Years - Case Report: Inoperable, functional relevant stenoses of the airways can be effectively treated short-termed and medium-termed by means of bronchoscopic stenting occasionally, silicone stents cause problems after long-term periods, such as migration and retention of bronchial secretions. Metallic stents can lead to obstructing granulomas or even bronchial wall perforation. As regards long-term treatment with airway stents over several years, there is little experience and no uniform recommendations are known. We report on a 76-year old female patient with a severe benign subglottic tracheostenosis after tracheostomy who was successfully treated by means of bronchoscopic dilatation and stenting with a silicone stent (POLYFLEX stent). POLYFLEX Stent is a self-expanding silicone stent with an encapsulated monofilament network made of polyester. The network is completely covered by a silicone layer with a smooth inner sur-

Pneumologie 2001; 55: $152-158$

(c) Georg Thieme Verlag Stuttgart · New York ISSN 0934-8387 face (protecting against incrustation) and a structured outer surface of the stent (protecting against migration). The ends of the monofilaments were provided with a special protection to avoid tissue granulation and to yield $x$-ray contrast. During a follow-up of almost 5 years the stent is well tolerated and there is no restenosis. Complications such as migration, obstructing secretions and obstructing granulomas did not occur. A slight bronchial hypersecretion presented no problem under regular inhalation therapy with isotonic $\mathrm{NaCl}$ solution. 21 and 56 months after stenting there were two episodes of minor haemoptysis. There was no demonstrable source of haemorrhage by bronchoscopy. After 56 months, biopsy at the distal opening of the stent showed a squamous cell metaplasia, but no granulation tissue. Microbiological analysis of bronchial secretions revealed an increasing, but clinically silent colonisation with potentially pathogenic microorganisms.

\section{Einleitung}

Therapie der Wahl von funktionell wirksamen benignen Stenosen der Trachea ist die Operation im Sinne einer Querresektion oder eines plastischen Eingriffes [1-5]. Bei lokaler oder funktioneller Inoperabilität, gelegentlich auch als Notfalleingriff bei akut dekompensierter Stenose ist die bronchoskopische Stenteinlage ein etabliertes Therapieverfahren. Bei benignen und bei malignen Stenosen sind prompte Besserungen stenosebedingter Beschwerden und lungenfunktioneller Befunde belegt [1,6-8]. Verschiedene Stenttypen sind auf dem Markt, die sich den 3 Gruppen Silikonstents, Metallstents und Hybridstents zuordnen lassen. Die Vor- und Nachteile der einzelnen Stents sind durch eine große Anzahl von Literaturmitteilungen in Form von Übersichten, Erfahrungsberichten und anekdotischen Mitteilungen bekannt [3,7-11]. Auch sind wichtige biomechanische Untersuchungen erfolgt [12]. Allerdings sind prospektive klinische Studien mit dem Ziel des Vergleichs der einzelnen Stentmodelle bis heute nicht publiziert worden, so dass keine allgemein gültigen Richtlinien zu deren Einsatz existieren [13]. Auch wurde bedauert, dass über den Langzeiteinsatz von Atemwegsstents über mehrere Jahre bislang kaum Erfahrungen vorliegen [14,15]. Tatsächlich finden sich auch in großen Serien nur vereinzelt Patienten mit einer Therapiedauer über 3 Jahre $[5,8,9,16-18]$.

Vor diesem Hintergrund ist es gerechtfertigt, die große Anzahl kasuistischer Mitteilungen zu diesem Thema um einen Fall zu 
erweitern, der sich durch eine Nachbeobachtungszeit von fast 5 Jahren auszeichnet.

\section{Kasuistik}

Patientin W. G., geb. 17.8.19. Als Kind erkrankte sie an rheumatischem Fieber mit rheumatischer Endokarditis, als Folge entwickelten sich eine Mitralinsuffizienz Grad II und eine Aorteninsuffizienz Grad I. Lebenslang bestand eine Bronchitisneigung. Zwischen 1940 und 1972 rauchte sie etwa 5 Zigaretten/d.

1972 wurde auswärtig ein Schilddrüsenkarzinom mit Thyreoidektomie und perkutaner Strahlentherapie behandelt. Präzise Angaben zu Histologie, Strahlendosis und Strahlenfeld sind nicht mehr verfügbar. Wegen postoperativer beidseitiger Recurrensparese wurde die Anlage eines Tracheostoma und die Versorgung mit einer Trachealkanüle erforderlich. 1980 konnte die Rückbildung der Recurrensparese belegt werden, so dass die Dekanülierung erfolgte und das Tracheostoma sich spontan verschloss.

In den nächsten Jahren war die respiratorische Situation zunächst stabil, es bestand jedoch Dyspnoe mit stridorösem Atmen bei mäßiger körperlicher Belastung.

Im Dezember 1994 kam es, begleitet von Symptomen eines fieberhaften Atemwegsinfektes, zu zunehmender, zuletzt massiver Atemnot und Stridor in Ruhe. In der Notfallambulanz eines auswärtigen Krankenhauses trat ein Atem- und Kreislaufstillstand ein. Die kardiopulmonale Reanimation war erfolgreich, allerdings musste bei einem Intubationshindernis in der oberen Trachea die Notfalltracheotomie durchgeführt werden.

Aus dem OP-Bericht: „Auf Höhe des ehemaligen Schilddrüsenlagers kein palpables Trachealrohr mit Knorpelringen, es findet sich derbes Knorpelgewebe. Auf Höhe des 3. Trachealringes Tracheotomie und Einlage einer 10er-Tracheoflexkanüle.“

Im Januar 1995 wurde auf Verlangen der Patientin gegen ärztlichen Rat die Trachealkanüle entfernt, das Tracheostoma verschloss sich erneut.

Am 7.3.1995 und 2.10.1995 stellte sich die Patientin ambulant beim Lungenfacharzt vor und klagte über erhebliche
Belastungsdyspnoe und Schwierigkeiten, Sekret abzuhusten. Klinisch bestand ein deutlicher in- und exspiratorischer Stridor. Lungenfunktionsuntersuchungen wurden von der Patientin verweigert, da forcierte Atemmanöver die Atemnot erheblich verschlechterten. Zu stationärer Diagnostik war die Patientin zunächst nicht bereit. Eine ambulante HNO-Untersuchung am 10.10.1995 zeigte beidseits mobile Stimmbänder, das rechte Stimmband war aber reduziert beweglich. Durch die Stimmritze hindurch zeigte sich eine hochgradige subglottische Trachealstenose.

Am 17.11.95 erfolgte die Einweisung in unsere Abteilung, nachdem in den letzten Tagen die Atemnot weiter zugenommen hatte, außerdem schon länger bestehende Schluckstörungen das Essen fester Speisen kaum noch erlaubten. Auf der Einweisung war vom Hausarzt vermerkt: „In- und exspiratorischer Stridor mit starker Atemnot. Gibt es eine Möglichkeit, die Trachealfunktion zu verbessern?“

Die Bedingungen von Seiten der Patientin lauteten: „Lieber sterbe ich, als mir nochmals eine Kanüle legen zu lassen. An meiner Luftröhre wird nichts mehr geschnitten!“

Klinischer Befund: $151 \mathrm{~cm}, 63 \mathrm{~kg}$, leicht reduzierter AZ, inund exspiratorischer Stridor mit Dyspnoe bei geringster Belastung. Prätibiale und Knöchelödeme. Über der Trachea ausgeprägt verschärftes Atemgeräusch, über allen Lungenfeldern mittel-grobblasige RG. Herz: RR 120/80, absolute Arrhythmie, bandförmiges Systolikum über Herzspitze. Abdomen o. B.

Die mit großen Schwierigkeiten durchgeführte Lungenfunktionsanalyse (Abb.1 u. Tab.1) zeigte in der Fluss-VolumenKurve eine hochgradige Einschränkung der forcierten in- und exspiratorischen Flussraten mit jeweils nahezu horizontalem Verlauf. Die Ganzkörperplethysmographie bot eine S-förmige

Tab. 1 Verlauf der Lungenfunktion

\begin{tabular}{lllll}
\hline Datum & $\begin{array}{l}11.1995 \\
\text { vor Stent }\end{array}$ & $\begin{array}{l}11.1995 \\
\text { nach Stent }\end{array}$ & 9.1997 & 7.2000 \\
\hline FEV 1 [I (\%soll)] & $1,2(63)$ & $1,6(84)$ & $1,5(82)$ & $1,4(79)$ \\
IVC [I (\%soll)] & $1,8(75)$ & $2,7(110)$ & $2,1(96)$ & $2,2(100)$ \\
$\begin{array}{l}\text { Raw tot } \\
\text { [kPa } \times \text { s/l (\%soll)] }\end{array}$ & $1,04(347)$ & $0,51(170)$ & $0,57(191)$ & $0,68(228)$ \\
\hline
\end{tabular}

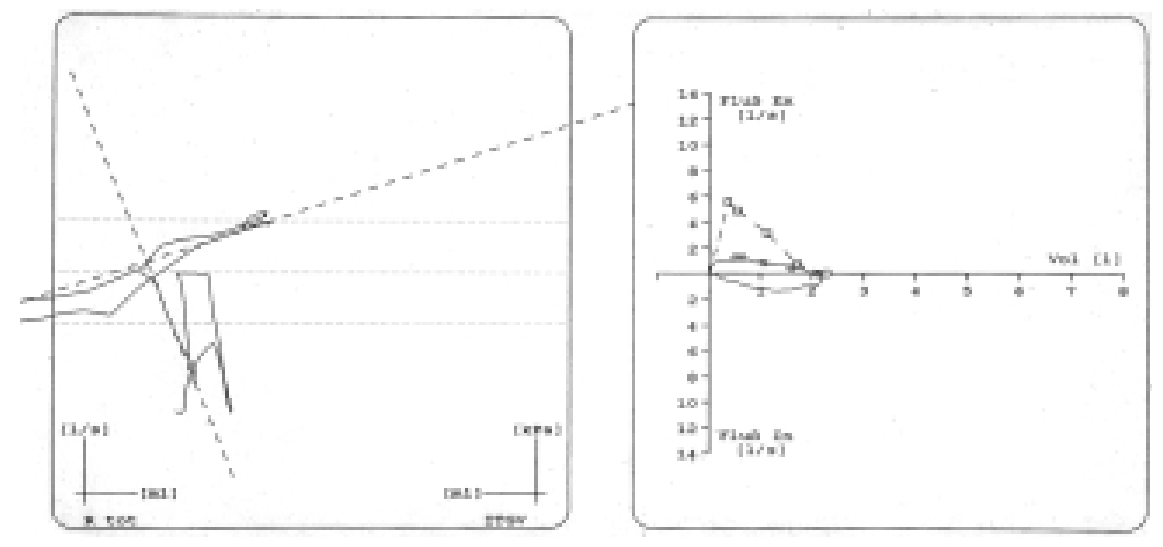

Abb. 1 Widerstands-Schleife und Fluss-Volumen-Kurve vor Stent. 
Deformierung der Fluss-Druckkurve. Das Verschlussdruckmanöver war nicht adäquat reproduzierbar. Insgesamt bestand eine schwere zentrale, kombiniert extra- und intrathorakale Obstruktion.

Die Fiberbronchoskopie in Lokalanästhesie zeigte die Stimmbänder beweglich mit stark reduziertem Bewegungsausmaß rechts. $3 \mathrm{~cm}$ subglottisch fand sich eine 80\%-ige Trachealstenose mit Wandeinziehung (Abb. 2). Das Restlumen war angedeutet schlitzförmig konfiguriert mit dorsoventralem Verlauf. Ein Passageversuch wurde unterlassen. In der mikrobiologischen Analyse des Bronchialsekretes zeigte sich kulturell kein Wachstum. Die CT des Halses demonstrierte subglottisch eine kurzstreckige Trachealstenose im Sinne einer Wandeinziehung. Komprimierendes Gewebe kam nicht zur Darstellung. Distal der Stenose fanden sich freie zentrale Atemwege. Das Ösophaguslumen war stark aufgeweitet (Abb. 3).

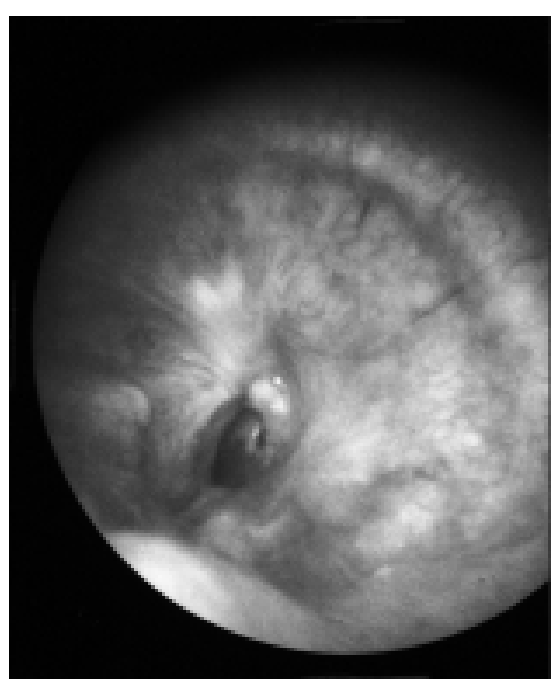

Abb. 2 Trachealstenose vor Stent Bronchoskopie.

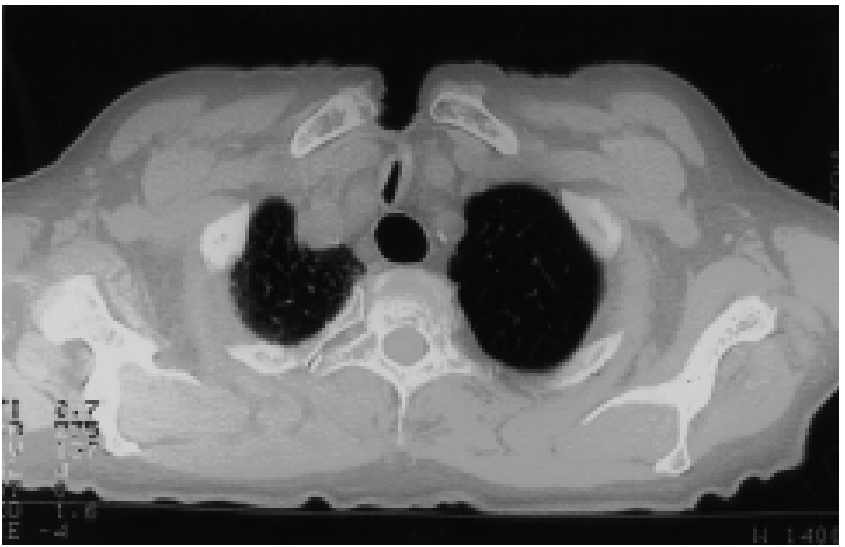

Abb. 3 Trachealstenose vor Stent - Computertomographie.

Durch Ösophagusbreischluck, Gastroskopie und Manometrie ergab sich die Diagnose einer Achalasie. Nach schrittweise durchgeführter Bougierung des Ösophagus bis $35 \mathrm{~mm}$ kam es zu einer erheblichen Besserung der Schluckbeschwerden, die respiratorische Situation blieb unverändert schlecht.
Diagnose: Komplexe subglottische Trachealstenose nach zweimaliger Tracheotomie und perkutaner Strahlentherapie.

\section{Therapie und Verlauf}

Die Patientin war mit dem Versuch der bronchoskopischen Dilatation der Trachealstenose einverstanden, lehnte aber jeden operativen Eingriff ab.

Bronchoskopie in Narkose mit starrem Instrument (7,5er STORZ1-Injekt-Bronchoskop): Mit mäßigem Druck konnte die Stenose unter rotierenden Bewegungen mit dem Bronchoskoprohr überwunden werden. Es resultierte jedoch kein bleibender Lumengewinn, da die Trachealwand bevorzugt links ventrolateral im Sinne einer Tracheomalazie nachgab. Daraufhin erfolgte die Einlage eines in unserem Bestand befindlichen Prototyps des POLYFLEX ${ }^{2}$-Stents (13 mm Innendurchmesser, $60 \mathrm{~mm}$ Länge) mit Hilfe des rot-kodierten EFERDUMON $^{3}$-Bronchoskopes. Es kam $\mathrm{zu}$ einer problemlosen Entfaltung des Stents, der die Stenose gut überbrückte und aufweitete.

Nach dem Eingriff verspürte die Patientin keinerlei Atemnot mehr, ein Stridor war nur noch bei forcierter Atmung wahrnehmbar. Die Lungenfunktion zeigte eine Normalisierung von Fluss-Volumenkurve und Ganzkörperplethysmographie (Abb. 4 u. Tab. 1).

Am 12.12.95 erfolgte die Entlassung. Zur Prophylaxe gegen Sekretinkrustation wurde eine Inhalationstherapie verordnet (3 mal täglich Inhalation von $5 \mathrm{ml}$ isotoner Kochsalzlösung mit Düsenvernebler [Pari-Boy $\left.\left.{ }^{4}\right]\right)$.

$\mathrm{Zu}$ weiteren, dringend empfohlenen Kontrollen hat sich die Patientin trotz wiederholter Kontaktaufnahmen bei Beschwerdefreiheit nicht bereit gefunden.

\section{9.97}

21 Monate später stellte sich die Patientin wegen blutiger Spuren im Auswurf ambulant vor. Nach ihren Angaben bestand keinerlei Atemnot, lediglich eine geringe Neigung zu bronchialer Verschleimung, die mit den Inhalationen jedoch gut zu beherrschen sei. Das Sekret sei von gelblicher Farbe und zäher Qualität. Schluckstörungen bestünden nicht. Klinisch war kein Stridor vernehmbar, die Lungenfunktion zeigte weiterhin einen Normalbefund (Tab.1).

Bronchoskopie flexibel in LA: Der Stent lag korrekt. Eine Migration, Sekretretention oder Sekretinkrustation bestanden nicht. Granulationsgewebe war auch im Bereich der Stentenden nicht erkennbar. Von links ventral war eine minimale, aber nicht kritische Einfaltung des Stents zu sehen. Zeichen einer aktuellen Blutung oder eine Blutungsquelle waren nicht darstellbar. Die mikrobiologische Analyse des Bronchialsekret zeigte mikroskopisch Granulozytenreichtum und kulturell Nachweis von E. coli (Tab. 2).

\footnotetext{
${ }^{1}$ Fa. Karl Storz GmbH, 78503 Tuttlingen

2 Willy Rüsch AG, 71394 Kernen

3 Bess GmbH, 14169 Berlin

4 Pari-Werk GmbH, 82317 Starnberg
} 

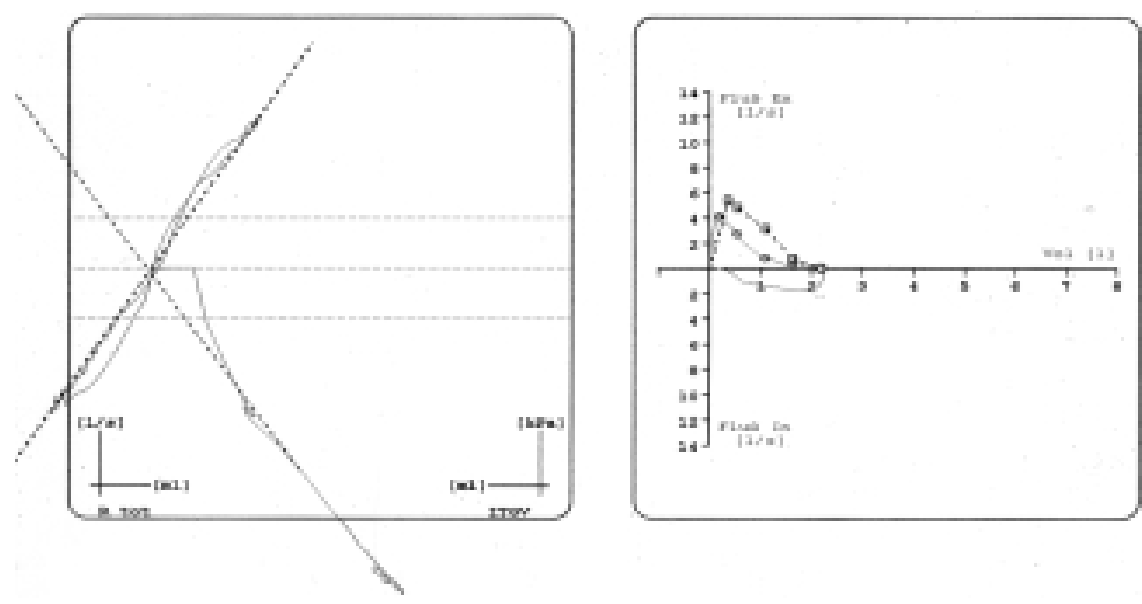

\begin{tabular}{|c|c|c|c|}
\hline Datum & 11.1995 & 9. 1997 & 7.2000 \\
\hline Granulozyten $\left(\times 10^{6} / \mathrm{ml}\right)$ & $<0,1$ & 1,5 & 0,2 \\
\hline $\begin{array}{l}\text { Mikrobiologische Kultur } \\
\left(\times 10^{6} / \mathrm{ml}\right)\end{array}$ & kein Wachstum & E. coli (5) & $\begin{array}{l}\text { Pseudomonas aeruginosa (500) } \\
\text { Staphylococcus epidermidis } \\
(500) \\
\text { Proteus vulgaris (100) }\end{array}$ \\
\hline
\end{tabular}

Abb. 4 Widerstands-Schleife und Fluss-Volumen-Kurve nach Stent.

Tab. 2 Verlauf der mikrobiologischen Befunde im Bronchialsekret
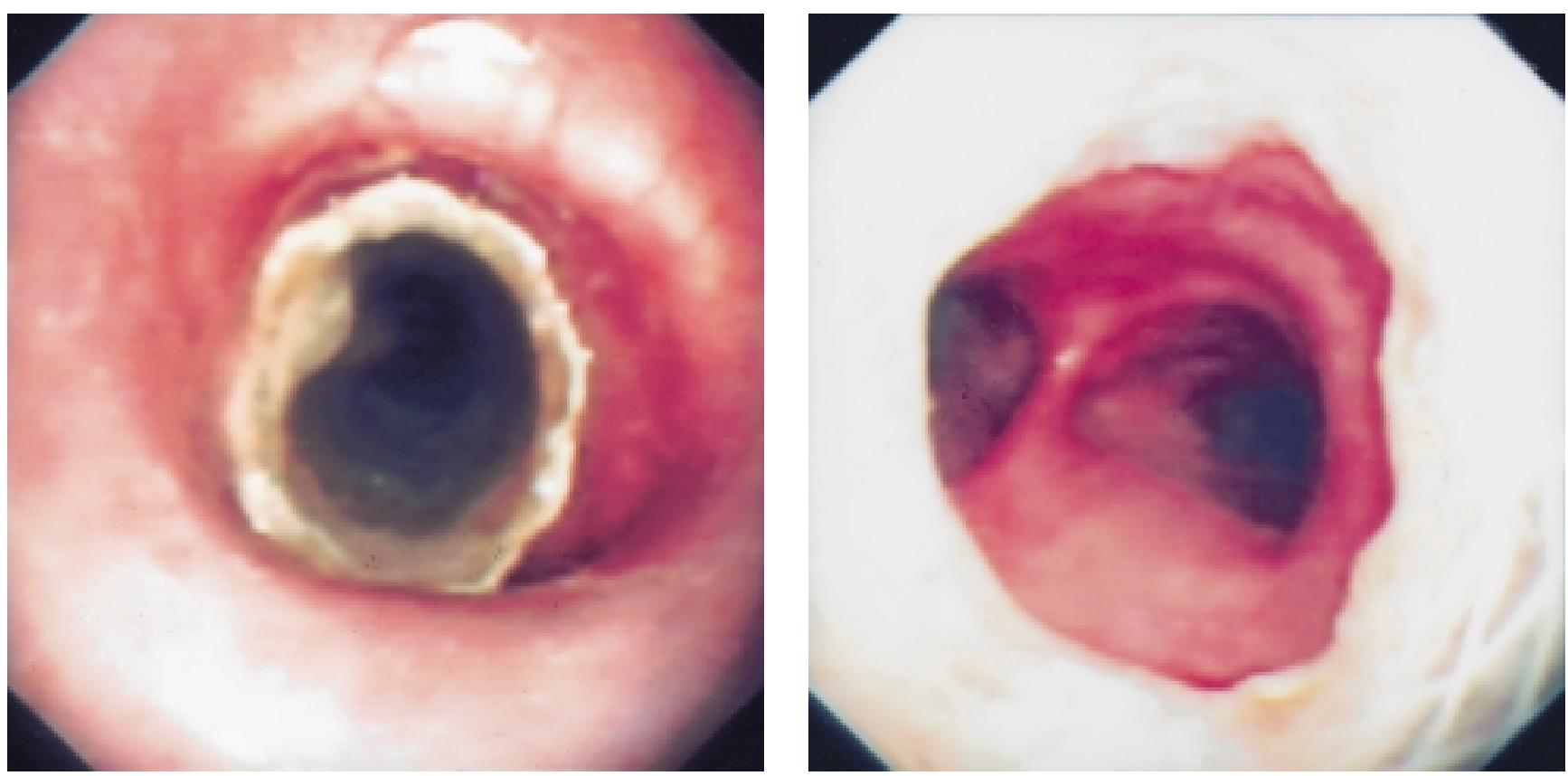

Abb. 5 u. 6 Bronchoskopie 56 Monate nach Stenteinlage - proximale und distale Stentöffnung.

Angesichts der günstigen Befunde und der subjektiven Beschwerdefreiheit wurden auch weiterhin keine Kontrolltermine wahrgenommen.

\section{7.00}

56 Monate nach Stenteinlage stellte sich die Patientin erneut wegen einzelner Blutpünktchen im Auswurf ambulant vor. Weiterhin bestanden weder Atemnot noch Schluckstörungen, unverändert war die regelmäßige Inhalation von isotoner $\mathrm{NaCl}$-Lösung die einzige bronchiale Therapie.

Klinisch und in der Lungenfunktion zeigten sich keine Veränderungen im Vergleich zu 1997 (Tab.1).

Bronchoskopie flexibel in LA: Sämtliche Befunde waren im Vergleich mit 1997 unverändert (Abb.5 u. 6). Lediglich im Bereich der distalen Stentöffnung imponierte an der Trachea- 

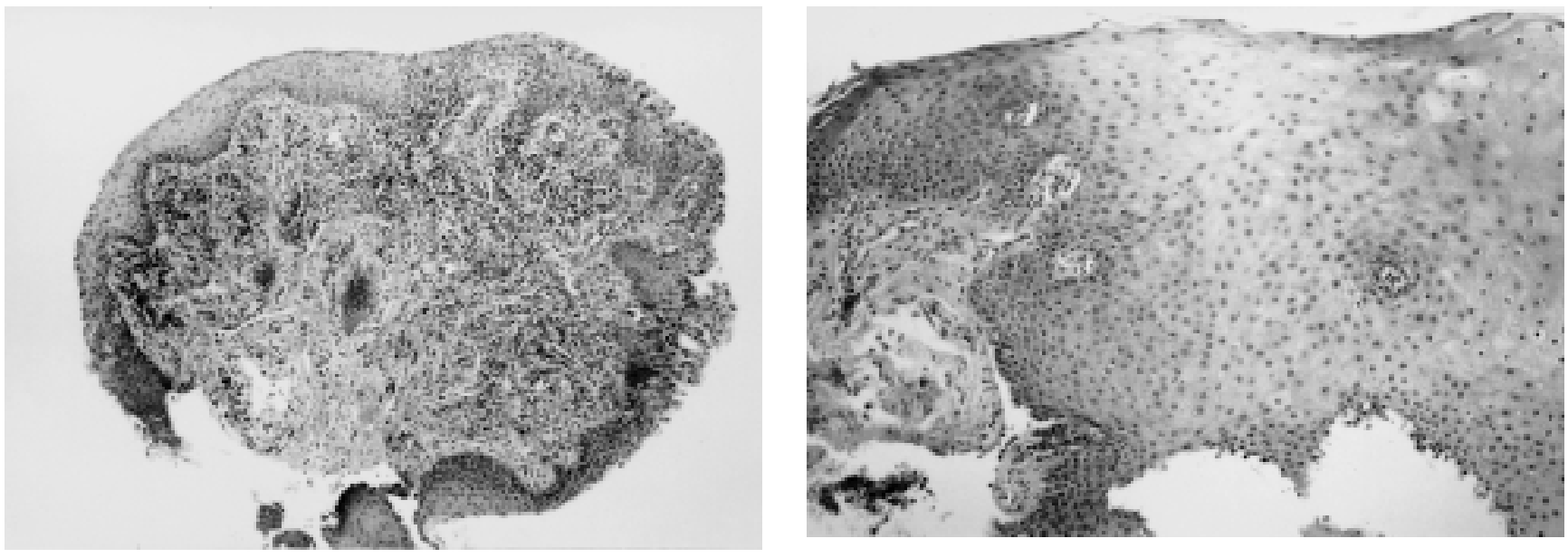

Abb. 7 u. 8 Biopsie aus Schleimhaut distale Stentöffnung (HE, × 100; HE, × 160). Chronische Entzündung mit Plattenepithelmetaplasie.

vorderwand in geringer Menge nicht blutendes, leicht polypöses Gewebe, welches biopsiert wurde. Die Stentinnenfläche war von einer dünnen Schicht leicht absaugbaren, gelblichen Sekretes belegt, das Stentlumen war aber weder stenosiert noch inkrustiert. Die histologische Untersuchung mehrerer Biopsate von der Tracheavorderwand zeigte Schleimhaut, teils mit Zylinderepithel mit Basalzellhyperplasie, teils mit metaplastischem Plattenepithel und einer chronischen Entzündung (Abb. 7 u. 8).

In der mikrobiologischen Analyse des Bronchialsekrets fanden sich mikroskopisch mäßig Granulozyten und kulturell verschiedene potenziell pathogene Keime (Tab. 2).

\section{9.00}

Anlässlich des 3. Deutschen Lungentages besuchte die Patientin eine unserer Veranstaltungen und berichtete über eine ungestörte Atmung.

\section{Diskussion}

Wir berichten über eine Patientin mit einer benignen komplexen subglottischen Trachealstenose, die sich nach Operation und perkutaner Strahlentherapie eines Schilddrüsenkarzinoms, nach zweimaliger Tracheotomie und mehrjähriger Trachealkanülierung entwickelt hatte. Dabei dürfte eher die Tracheotomie als die Strahlentherapie für die Ausbildung der Trachealstenose verantwortlich gewesen sein, da eine Strahlentherapie der Halsregion nur sehr selten zu Trachealstenosen führt [3], diese Komplikation dagegen insbesondere bei chirurgischen Tracheotomien häufig auftritt [19]. Begleitend litt die Patientin an einer Achalasie. Da die Induktion einer funktionell wirksamen Trachealstenose alleine auf dem Boden einer Achalasie beschrieben ist [20], erfolgte zunächst eine Bougierung des Ösophagus. Doch auch die erfolgreiche Behandlung der Achalasie besserte die Atemnot nicht.

Vor dem Hintergrund, dass die Patientin die klassische Therapie in Form eines operativen Eingriffes strikt ablehnte und dass aufgrund der hochgradigen respiratorischen Einschränkung ein dringender Therapiebedarf bestand, wurde eine Dilatation der Stenose durchgeführt. Diese Strategie ist bei inoperablen Trachealstenosen ein etabliertes Verfahren, wobei man mit Bougies, Ballondilatatoren oder - wie in unserem Fall - mit dem Rohr des starren Bronchoskopes arbeiten kann $[2,4]$. Da es sich schon während der Dilatation zeigte, dass keine bleibende Lumenerweiterung resultierte, wurde ein Trachealstent eingelegt. Prinzipiell bestand bei unserer Patientin hinsichtlich einer Stenteinlage eine komplizierte Situation. Allgemein sind die Behandlungsergebnisse bei subglottischer Lage von Trachealstenosen häufig unbefriedigend [2,5], zum anderen werden bei benigner Stenose wegen der zu erwartenden Langzeittherapie an den Stent besondere Anforderungen hinsichtlich Funktionssicherheit und Gewebeverträglichkeit gestellt. Kunststoffstents sind zwar gewebeverträglich, zeigen im Langzeiteinsatz aber häufig die Probleme Stentdislokation, Sekretretention und Sekretinkrustation $[3,5,11,18]$. Die Sekretretention ist insbesondere dann zu befürchten, wenn, wie bei unserer Patientin, eine Hustenschwäche durch Recurrensteilparese besteht [18]. Metallstents werden langfristig von der Trachealwand oft schlecht vertragen, mit Granulationsgewebsbildung oder sogar Wandperforation muss gerechnet werden $[1,9,10]$.

Wir entschieden uns bei unserer Patientin für die Einlage des POLYFLEX-Stents, weil sich bei der Bronchoskopie zeigte, dass dieser Stent die Trachealstenose problemlos aufweitete, aufgrund seiner dünnen Wandung das Tracheallumen nur gering reduzierte und sich zudem der Konfiguration der Stenose ohne wesentliche Deformierung anpasste. Bei dem von uns verwendeten Modell handelt es sich um einen Prototypen, der jedoch bis auf fehlende Röntgenkontrastmarkierungen der Stentöffnungen und in die Wand eingearbeitete Röntgenkontrastpunkte mit dem derzeit im Handel befindlichen POLYFLEX-Stent identisch ist. Der POLYFLEX-Stent ist ein selbstexpandierender Silikonstent aus einem Geflecht von Polyestermonofilamenten. Das Geflecht ist vollständig in einer Silikonkautschukschicht eingebettet. Die Innenfläche ist zum Schutz gegen Sekretinkrustation glatt, die Außenfläche zur Vermeidung von Migration konturiert. Bei der biomechanischen Analyse zeigt der POLYFLEX-Stent eine lineare Kompressionsdruck-Rückstellkraft-Kurve, ähnlich wie der Silikonstent nach DUMON. Anders als dieser kann sich der POLY- 
FLEX-Stent aber, wie in unserem Fall, der Form unregelmäßiger Stenosen, etwa Sanduhrstenosen, anpassen [12]. Viele Erfahrungen über die Langzeitverträglichkeit des POLYFLEX-Stents liegen nicht vor [14]. Bei Lungentransplantationen wurde er zur Prophylaxe bronchialer Anastomosenstenosen über maximal 12 Monate mit Erfolg eingesetzt. Bei einer Gruppe von 7 Patienten wurde einmal eine Migration und einmal eine Sekretretention beobachtet [21]. Im Tierexperiment bei Schafen wurde über ein Jahr eine problemlose Gewebeverträglichkeit gesehen [22]. Der heute übliche spezielle Kunststoffapplikator zur Einlage des POLYFLEX-Stents war 1995 noch nicht verfügbar, so dass der Metallapplikator des EFER-DUMON-Bronchoskops verwendet wurde, was ohne Schwierigkeiten möglich war. Allerdings muss man darauf achten, den Stent bei der Einfaltung in das Metallrohr nicht zu beschädigen.

Bei unserer Patientin zeigte sich direkt nach Stenteinlage eine völlige Rückbildung der zuvor bestehenden schweren zentralen Obstruktion, Atemnot bestand nicht mehr. Selbstverständlich wären engmaschige regelmäßige Nachuntersuchungen mit Lungenfunktionsanalyse und Bronchoskopie unbedingt erforderlich gewesen $[1,5]$, doch scheiterte dies an der bei subjektiver Beschwerdefreiheit fehlenden Bereitschaft der Patientin. Innerhalb von knapp 5 Jahren waren lediglich 2 Nachuntersuchungen möglich, die jeweils aufgrund von leichten Hämoptysen durchgeführt wurden. Zu beiden Zeitpunkten konnte eine unauffällige Ventilation und eine korrekte Stentfunktion belegt werden. Eine Stentmigration trat nicht auf, zu einer klinisch relevanten Sekretretention oder gar Sekretinkrustation kam es unter einer regelmäßigen Inhalationstherapie mit isotoner Kochsalzlösung über einen Düsenvernebler nicht.

Die mikrobiologische Analyse des Bronchialsekretes zeigte zwischen 1995 und 2000 zunehmend potenziell pathogene Keime, obwohl klinisch, radiologisch und im Labor jeweils keine Hinweise auf eine Infektanfälligkeit bestanden. Somit ist eher von einer bronchialen Besiedelung als von einer bronchialen Infektion auszugehen. Das Phänomen der bronchialen Besiedelung mit potenziell pathogenen Keimen ohne Zeichen der Infektion bei Trägern von Kunststoffstents wurde schon von anderen Autoren mitgeteilt und nicht als Indikation für eine antibiotische Therapie angesehen. Als Ursache wird eine Beeinträchtigung der mukoziliären Klärfunktion durch den Stent vermutet $[18,23]$.

56 Monate nach Stenteinlage war im Bereich des aboralen Stentendes ventral in geringer Menge nicht obstruierendes, polypöses Gewebe zu sehen. Die histologische Untersuchung zeigte Schleimhaut mit chronischer Entzündung und Plattenepithelmetaplasie. Die Bildung von Granulationsgewebe, welche bevorzugt Bereich der Stentenden auch bei Kunststoffstents gelegentlich beobachtet wird $[5,18]$, war bei unserer Patientin nicht nachweisbar. Zusammenfassend war bei unserer Patientin mit einer hochgradigen benignen subglottischen Trachealstenose auf dem Boden zweier Tracheotomien die Therapie mit bronchoskopischer Bougierung und Einlage eines POLYFLEX-Stents erfolgreich. Über einen Beobachtungszeitraum von bislang fast 5 Jahren kam es zu keinen gravierenden Problemen im Sinne von Rezidivstenose, Stentmigration, Sekretinkrustation oder lokalen geweblichen Unverträglichkeitsreaktionen. Wir beobachteten eine erschwerte spontane Bronchialsekretmobilisation, die eine regelmäßige Inhalationstherapie erforderlich machte. Eine interventionspflichtige Sekretretention bestand zu keiner Zeit. Zwei Episoden mit leichten Hämoptysen ohne bronchoskopischen Nachweis einer Blutungsquelle, eine nicht stenosierende Plattenepithelmetaplasie am aboralen Stentende und eine klinisch nicht symptomatische Besiedelung des Bronchialsekretes mit potenziell pathogenen Keimen sind wahrscheinlich auf die Stenteinlage zurückzuführen, ohne bislang den Therapieerfolg zu gefährden.

\section{Literatur}

${ }^{1}$ Bolliger CT. Prosthesis: indications, contraindications and follow-up. Rev Mal Respir 1999; 16: 665-672

2 Pearson FG. Technique of management of subglottic stenosis. Chest Surg Clin N Am 1996; 6: 683-692

${ }^{3}$ Colt H, Mathur P. Airway stents. Up to date, 1999: Vol. 8 Nr. 2

${ }^{4}$ Freitag L. Tracheobronchiale Stents - welcher Stent für welche Indikation?. Atemw.-Lungenkrkh 1995; 12: 609-614

${ }^{5}$ Brichet A, Verkindre C, Dupont J, Carlier ML, Darras J, Wurtz A, Ramon P, Marquette $\mathrm{CH}$. Multidisciplinary approach to management of postintubation tracheal stenoses. Eur Respir J 1999; 13: $888-893$

${ }^{6}$ Stohr S, Bolliger CT. Stents in the management of malignant airway obstruction. Monaldi Arch Chest Dis 1999; 54: 264-268

${ }^{7}$ Cavaliere S, Venuta F, Foccoli P, Toninelli C, La Face B. Endoscopic treatment of malignant airway obstruction in 2008 patients. Chest 1996; 110: 1536-1542

${ }^{8}$ Dassgupta A, Dolmatch BL, Abi-Saleh WJ, Mathur PN, Mehta AC. Self expandable metallic airway stent insertion employing flexible bronchoscopy: preliminary results. Chest 1998; 114: 106-109

${ }^{9}$ Filler RM, Forte V, Chait P. Tracheobronchial stenting for the treatment of airway obstruction. J Pediatr Surg 1998; 33: $304-$ 311

${ }^{10}$ Hramiec JE, Haasler GB. Tracheal wire stent complication in malacia: implications of position and design. Ann Thorac Surg 1997; 63: 209-212

${ }^{11}$ Noppen M, Meysman M, Claes I, D’Haese J, Vinken W. Screwthread vs Dumon endoprosthesis in the management of tracheal stenosis. Chest 1999; 115: 532-535

${ }^{12}$ Freitag L. Biomechanik von Atemwegsstenosen und Stents. Atemw.-Lungenkrkh 1997; 23: 194-200

${ }^{13}$ Mehta AC, Dasgupta A. Airway stents. Clin Chest Med 1999; 20: 139- 151

${ }^{14}$ Bolliger CT, Weyser C, Wu X, Hauser R, Studer W, Dalquen P, Perruchoud AP. Evaluation of a new self-expandable silicone stent in an experimental tracheal stenosis. Chest 1999; 115: 496-501

${ }^{15}$ Colt HG, Dumon JF. Airway stents. Present and future. Clin Chest Med 1995; 16: 465-478

${ }^{16}$ Stephens Jr KE, Wood DE. Bronchoscopic management of central airway obstruction. J Thorac Cardiovasc Surg 2000; 119: 289296

${ }^{17}$ Neville WE, Bolanowski JP, Kotia GG. Clinical experience with the silicone tracheal prosthesis. J Thorac Cardiovasc Surg 1990; 99: 604-612

${ }^{18}$ Freitag L, Tekolf E, Stamatis G, Greschuchna D. Clinical evaluation of a new bifurcated dynamic airway stent: a 5-year experience with 135 patients. Thorac Cardiovasc Surgeon 1997; 45: $6-12$

${ }^{19}$ Rumbak MJ, Walsh FW, Anderson WM, Rolfe MW, Solomon DA. Significant tracheal obstruction causing failure to wean in patients requiring prolonged mechanical ventilation: a forgotten 
complication of long-term mechanical ventilation. Chest 1999; 115: 1092 - 1095

${ }^{20}$ Berrisford RG, Oo A, Walshaw MJ, Drakeley MJ. Tracheal obstruction in achalasia: a role for airway stenting?. Ann Thorac Surg 1998; 66: 939-941

${ }^{21}$ Ewert R, Waurick P, Wenig Y, Grauhan O, Hummel M, Hetzer R. Intraoperative placement of a new silicone stent $\left(\right.$ POLYFLEX $\left.^{\circledR}\right)$ in lungtransplanted patients - a new application of bronchial stents. Eur Respir J 1997; 10, Suppl. 25: 369s - P2346

22 Puma F, Farabi R, Urbani M, Santoprete S, Daddi N, Di Meo A, Gialetti R, Tocchi A, Daddi G. Long-term safety and tolerance of silicone and self-expandable airway stents: an experimental study. Ann Thorac Surg 2000; 69: 1030-1034

${ }^{23}$ Noppen M, Pierard D, Meysman M, Claes I, Vincken W. Bacterial colonization of central airways after stenting. Am J Respir Crit Care Med 1999; 160: 672-677

\section{Dr. med. J. Schildge}

St. Vincentius-Krankenhäuser Karlsruhe

Medizinische Klinik - Abteilung Pneumologie

Südendstraße 32

76137 Karlsruhe

E-mail: Johannes.Schildge@t-online.de

Internet: www.vincentius-ka.de

\section{BUCHBESPRECHUNG}

W. Petro (Hrsg.): Pneumologische Prävention und Rehabilitation. Ziele - Methoden - Ergebnisse. 2. Aufl. SpringerVerlag Berlin, Heidelberg 2000. 721 S. , 130 Abb. Kart. DM 246,-. ISBN 3-540-67113-70.

Unter der Mitarbeit zahlreicher Rehabilitationsmediziner aus Klinik und Praxis ist wiederum ein umfassendes Lehrbuch entstanden, das die moderne pneumologische Rehabilitation unter die Prämisse eines patientenorientierten, ganzheitlichen methodischen Ansatzes stellt

Prägnant schildert der erste Teil die Historie präventiven und rehabilitativen Denkens und Handelns bis zu den heutigen Rechtsgrundlagen. Deutlich wird vor allem, dass der Wandel im Panorama der Krankheiten zu den chronischen und verhaltensabhängigen Erkrankungen gerade in der Pneumologie eine angemessene Diagnostik und Therapie unter Einschluss präventiver und edukativer Maßnahmen nach sich ziehen muss. Dieser Ansatz erfordert zudem eine interdisziplinäre Teamarbeit zwischen Ärzten, Therapeuten, Psychologen und Sozialarbeitern.

Der zweite Teil ist der pneumologischen Prävention gewidmet und beschäftigt sich mit Definitionen sowie Strategien im stationären und ambulanten Bereich. Das enge Ineinandergreifen von Prävention und Rehabilitation deutet sich hier schon an, gelten doch z.B. stationäre rehabilitative Maßnahmen wie Patientenverhaltenstraining oder Sporttherapie ebenso als Tertiärprävention und können darüber hinaus ambulant weiter geführt werden. In einem eigenen Kapitel werden präventive Maßnahmen in der Pädiatrie behandelt.

Den weitaus größten Teil des Buches nimmt schließlich das Kapitel „Pneumologische Rehabilitation“ ein. Die Einführung schildert Definitionen und Zielsetzungen in bezug auf die stationäre und ambulante Rehabilitation, wobei letztere zur Zeit in Deutsch- land auf einzelne Elemente (Schulung, Asthmasport) sowie Modelle beschränkt bleibt. Weiterhin zeigt sich die Vernetzung von pneumologischer Rehabilitation zu vor- und nachstationären Maßnahmen ausgesprochen defizitär.

Auf den theoretischen Teil folgen Ausführungen zu Epidemiologie, Klinik und Diagnostik der klassischen chronischen Lungenerkrankungen, die dem Rehabilitationsmediziner begegnen. Gerade bei den chronisch-obstruktiven Lungenerkrankungen finden sich hier Informationsüberschneidungen, die aufgrund der Vielzahl der Autoren sicher nicht zu vermeiden sind.

Ausführlich werden alle in der pneumologischen Rehabilitationsmedizin erforderlichen diagnostischen Maßnahmen behandelt, darunter im besonderen die pulmonale und kardiale Funktionsdiagnostik, die Beurteilung der körperlichen Leistungsfähigkeit sowie die Allergiediagnostik. Berücksichtigt werden darüber hinaus auch die Schlafapnoe und die Chronobiologie.

Knapp 300 Seiten füllt die Darlegung der in der pneumologischen Rehabilitation gebräuchlichen Therapiemaßnahmen. Neben der medikamentösen Therapie sind dies vor allem die physikalische und die Klimatherapie sowie das körperliche Training und das Patientenverhaltenstraining. Gerade die Kapitel über medizinische Trainingstherapie und Patientenverhaltenstraining bei obstruktiven Atemwegserkrankungen Erwachsener sind sehr informativ geschrieben und vermitteln einen hervorragenden Überblick über Inhalte und Effekte. Weitere Kapitel beschäftigen sich mit der Rehabilitation atemwegserkrankter Kinder und Jugendlicher, mit der Raucherentwöhnung und mit psychotherapeutischen Behandlungsmöglichkeiten.

Im Anschluss an den Therapie-Teil geben verschiedene Autoren einen Überblick über die sozial- und arbeitsmedizinische Begutachtung und über rechtliche Grundlagen der Rehabilitation. Die letzten Kapitel beschäftigen sich mit der Beurteilung der Effektivität medizinischer Rehabilitationsmaßnahmen, u.a. mit der Auswahl geeigneter Messinstrumente sowie mit dem Begriff der Lebensqualität als wichtigem Gesundheitsindikator für den Rehabilitationserfolg.

$\mathrm{Zu}$ begrüßen ist, dass jedes Kapitel mit einem Literaturverzeichnis abschließt, das den tieferen Einstieg in das jeweilige Thema erleichtert. Die durchgehend identische Tabellengestaltung und eine Reihe von informativen Bilddokumenten tragen sehr zum Verständnis bei.

Insgesamt ist das vorliegende Werk ein wertvoller Leitfaden sowohl für alle an der Versorgung pneumologischer - erwachsener und pädiatrischer - Patienten Beteiligte in Klinik und Praxis als auch für ausgewiesene pneumologische Rehabilitationsmediziner. M. Behnke, Großhansdorf 